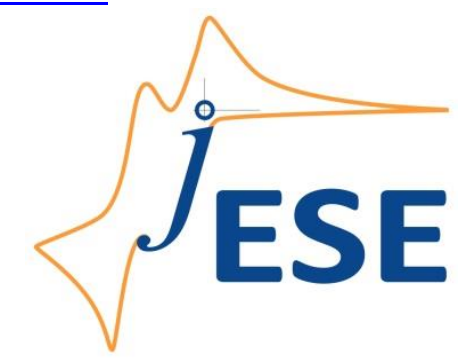

Open Access: ISSN 1847-9286

www.jESE-online.org

Original scientific paper

\title{
Laser induced selective electroless copper plating on polyurethane using EDTA-Cu as active material
}

\author{
Binggong Yan, Xiaodi Huang, Xuan Song, Lei Kang, Qihe Le, Jiang Kaiyong ${ }^{\bowtie}$ \\ Fujian Key Laboratory of Special Energy Manufacturing, Xiamen Key Laboratory of Digital Vision \\ Measurement, Huaqiao University, Xiamen 361021, China
}

Corresponding authors E-mail: 『jiangky@hqu.edu.cn

Received: June27, 2018; Revised: August 10, 2018; Accepted: August 11, 2018

\begin{abstract}
Using EDTA-Cu as the active material and polyurethane as the matrix, flexible cathodes were fabricated by laser-induced electroless copper plating process (LPKF-LDS) and characterized by SEM, X-ray energy spectrum and Auger electron spectroscopy. Flexible cathodes prepared from EDTA-CU and polyurethane showed good selectivity in copper plating process. Composition and particle morphology of EDTA-Cu, laser power, scanning speed, laser wavelength, laser spot size, pulse frequency etc. are the main factors that affect the fineness of electroless copper plating. By adjusting these parameters, the fineness of the copper plating was improved. $X$-ray energy spectrum and Auger electron spectroscopy results showed that after the laser scanning, both $\mathrm{Cu}^{0}$ and $\mathrm{Cu}^{+}$appeared in the scanning area, revealing thus the mechanism of electroless copper plating for polyurethane-EDTA-Cu flexible cathodes.
\end{abstract}

\section{Keywords}

EDTA-Cu, polyurethane, LPKF-LDS, flexible cathode

\section{Introduction}

Laser-induced electroless copper plating process (LPKF-LDS) is an innovative method for printing circuit on a polymer and its use in fabrication of a flexible cathode for electrochemical machining (FCEM) has also been expected [1]. Electrochemical machining using FCEM can make preparation of textures on freeform surfaces easier than a traditional process and it effectively reduces time and money costs due to FCEM reusability [2,3]. The main problem of FCEM is the fineness of the electroless copper plating, which is associated with properties of active material and the size of laser heat affected zone. Therefore, choosing of suitable active materials, reducing their particle size, increasing the uniformity of particles and minimizing the laser heat affected zone, are the main ways to improve the fineness of FCEM. In recent years, a variety of materials has been used as active 
materials to prepare FCEMs, such as palladium oxide [4-7], copper aluminate [8] and carbon nanotubes [9-12]. These materials, however, showed different processing problems that limited development of FCEMs. For example, the cost of palladium is too high to be suitable for industrial production, the manufacturing process of copper aluminate is complicated, and multi-wall carbon nanotubes related flexible cathodes are brittle and fragile. Therefore, new active materials need to be developed to resolve these problems. Based on the special copper based composite material, our group has already carried out some systemic studies on FCEM [13-15]. Owing to poor dispersion and easy agglomeration of the particles, however, the selectivity of electroless copper plating and the flexibility of cathode were found still unsatisfactory.

Considering the present researching progress on FCEMs, here, we propose application of EDTA$\mathrm{Cu}$ related flexible cathode for FCEM. EDTA-Cu is a chelated complex formed between ethylene diamine tetraacetic acid (EDTA) and copper. EDTA is a representative material of chelating agents and can form stable water-soluble complexes with alkali metals, rare earth elements and transition metals. Also, it is commonly used in the sewage treatment and can be applied to titrate nickel, copper, etc. EDTA-Cu is a low cost and easy to be prepared stable metal complex. In this paper, EDTA-Cu is used as the active material and polyurethane as the matrix for flexible cathodes that were fabricated and characterized.

\section{Experimental}

\section{Preparation of EDTA-Cu}

Considering the molar ratio of $\mathrm{Cu}: \mathrm{EDTA}=1: 1,2.67 \mathrm{~g}$ copper acetate monohydrate, $\left(\mathrm{CH}_{3} \mathrm{COO}\right)_{2} \mathrm{Cu} \cdot \mathrm{H}_{2} \mathrm{O}$ (99\%, Aladdin) and $3.9 \mathrm{~g}$ ethylene diamine tetraacetic acid, EDTA (99\%, Aladdin) were dissolved in $100 \mathrm{~mL}$ deionized water. The solution was refluxed at $100{ }^{\circ} \mathrm{C}$ for 2 hours using magnetic stirrer (ZNCL-B, Gongyi Yihua Instrument Co., Ltd.). The resultant was poured into ethanol and suction filtered for three times using a glass filter to remove $\mathrm{CH}_{3} \mathrm{COOH}$. The obtained powder was dissolved in deionized water and sprayed by a closed spray dryer (YC-501, Shanghai Yage Instrument Equipment Co., Ltd.).

\section{Fabrication of flexible cathode}

EDTA-Cu powder was dried in a vacuum oven at $70{ }^{\circ} \mathrm{C}$ for 2 hours, and then mixed according to the mass ratio of polyurethane : EDTA-Cu $=85: 15$. The resultant mixture was stirred thoroughly with a solder paste stirrer (ZB500S), after which an antifoaming agent ( 3 wt.\%, W-160, Zhongwan Co., Ltd.) was added and vibrated by ultrasonic curing [16] for 24 hours. Finally, the obtained modified polyurethane solution was smoothly poured onto the glass panel to get the polyurethane sheet with a thickness of $1 \mathrm{~mm}$.

\section{Laser activation}

A normal laser marking machine with a wavelength of $1064 \mathrm{~nm}$, a picosecond laser marking machine with a wavelength of $1064 \mathrm{~nm}$ and a UV laser marking machine with a wavelength of $355 \mathrm{~nm}$ were used to scan the EDTA-Cu modified polyurethane. The parameters of laser scanning process used in the experiments were as follows: pulse repetition frequency $1000 \mathrm{kHz}$, scanning speed $0.1 \mathrm{~m} / \mathrm{s}$, spot mode TEMOO.

\section{Electroless copper plating}

$1.6 \mathrm{~g}$ copper sulfate was fully dissolved in $25 \mathrm{~mL}$ of deionized water and complexing agents ( $1.4 \mathrm{~g}$ $\mathrm{C}_{4} \mathrm{O}_{6} \mathrm{H}_{4} \mathrm{KNa}$ and $2.5 \mathrm{~g}$ EDTA-2Na) were added to the copper sulfate solution to form a complex of 
copper ions. After that, a sodium hydroxide solution $(1.4 \mathrm{~g}$ sodium hydroxide, $25 \mathrm{~mL}$ deionized water) was added to the copper sulfate solution. Finally, the stabilizer ( $0.8 \mathrm{mg} 2,2^{\prime}$-Bipyridyl and $\left.7 \mathrm{mg} \mathrm{K}{ }_{4} \mathrm{Fe}(\mathrm{CN})_{6} \cdot 3 \mathrm{H}_{2} \mathrm{O}\right)$, reductant $(1.2 \mathrm{~mL} \mathrm{HCHO})$ and deionized water were added to obtain $100 \mathrm{~mL}$ of electroless copper plating solution. The laser activated modified polyurethane substrate was sonicated in $10 \%$ sodium hydroxide solution to clean the oil, rinsed with deionized water, and then kept in an oven at $80^{\circ} \mathrm{C}$ for 2 hours. The EDTA-Cu modified polyurethane sheet was immersed in the electroless copper plating solution at $80^{\circ} \mathrm{C}$ for 1 hour and then rinsed with deionized water.

\section{Characterization}

Surface morphologies of plated coatings were observed using a built-in spectrometer benchtop scanning electron microscope (Phenom). Adhesion between plated copper and polyurethane was tested by the scotch tape that was produced by 3M according to ASTM-D3359-09. The resistivity of the plated copper was measured by 4-probe method using SX1934(SZ-82). The surface of the substrate before and after the laser activation was characterized by XPS (Thermo escalab 250Xi). Before characterization, the surface of the sample was etched with an Ar particles beam having an etching depth of $5 \mathrm{~nm}$ to eliminate the effect of surface contamination on the characterization results. XPS photoelectron spectroscopy of the scanned area and Auger electron energy spectrum XAES were used to characterize the valence of copper on the surface of the substrate.

\section{Electrochemical machining}

Electrochemical machining was carried out using EDTA-Cu modified polyurethane as cathode, $5 \%$ sodium nitrate solution (with deionized water as solvent) as electrolyte and galvanized iron sheet as anode. Processing parameters were as follows: voltage $15 \mathrm{~V}$, pulse frequency $5 \mathrm{kHz}$ and duty ratio $50 \%$.

\section{Results and discussion}

\section{Morphology of EDTA-Cu}

During the preparing process of EDTA- $\mathrm{Cu}, \mathrm{CH}_{3} \mathrm{COOH}$ is generated from the reaction between EDTA and $\left(\mathrm{CH}_{3} \mathrm{COO}\right)_{2} \mathrm{Cu} \cdot \mathrm{H}_{2} \mathrm{O}$, playing a negative role in the electroless copper plating process. Figure 1 a shows the surface morphology of the coating with $\mathrm{CH}_{3} \mathrm{COOH}$ remaining at the surface.

Many voids can be seen and the edge of the plated copper line that is not clear. The main reason for this phenomenon could be that $\mathrm{CH}_{3} \mathrm{COOH}$ dissolved in the copper plating solution resulted in many voids on the top surface of the polyurethane, and copper particles are deposited around the voids by microporous adsorption. In order to eliminate the influence of $\mathrm{CH}_{3} \mathrm{COOH}$, as-prepared EDTA-Cu was dissolved in the ethanol and suction filtered for several times using a sand core filter device. Figure $1 \mathrm{~b}$ shows the morphology of the coating after removing $\mathrm{CH}_{3} \mathrm{COOH}$, where the quality of the coating is found significantly improved. However, the continuity of copper lines is still not good. Poor continuity observed in Figures $1 \mathrm{a}$ and $1 \mathrm{~b}$ resulted from irregular particle morphology and uneven sizes. In order to obtain EDTA-Cu with uniform particles, the suction filtered EDTA-Cu powder was dispersed in deionized water and spray dried. The morphology of spray-dried EDTA-Cu particles is shown in Figure 2a, where it can be seen that spray-dried EDTA-Cu particles are nearly spherical in shape and uniform in size. 


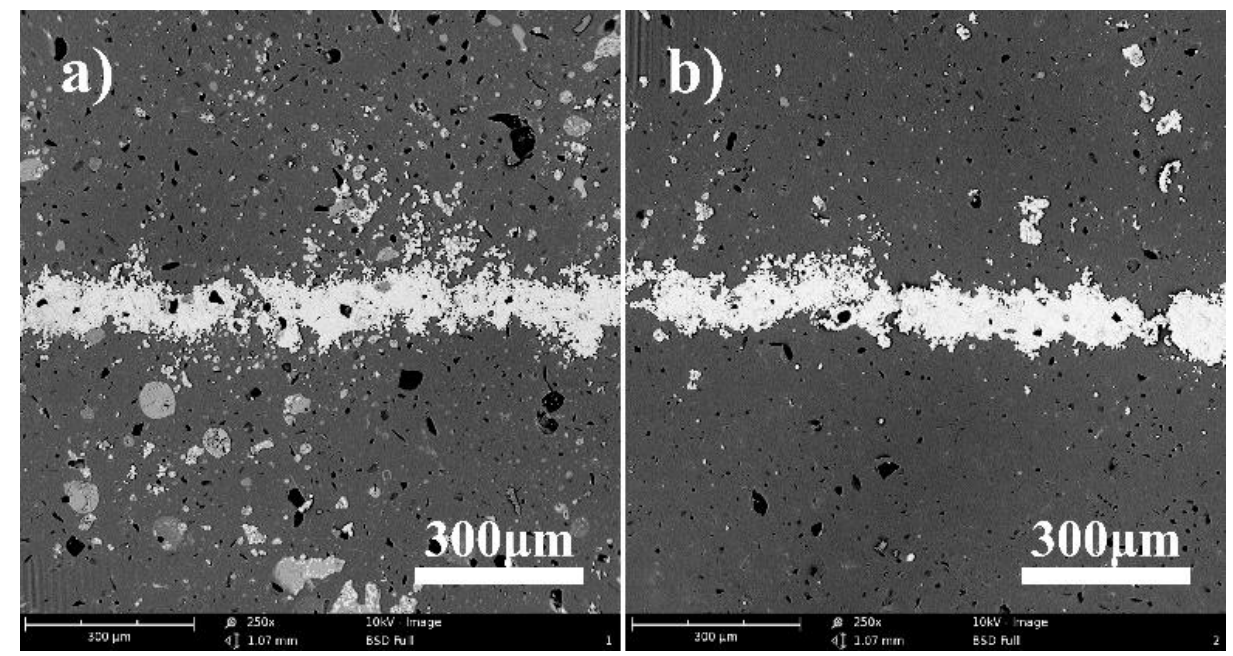

Figure 1. Surface morphology of flexible cathode with active materials: a) as-prepared EDTA-Cu, b) suction filtered EDTA-Cu
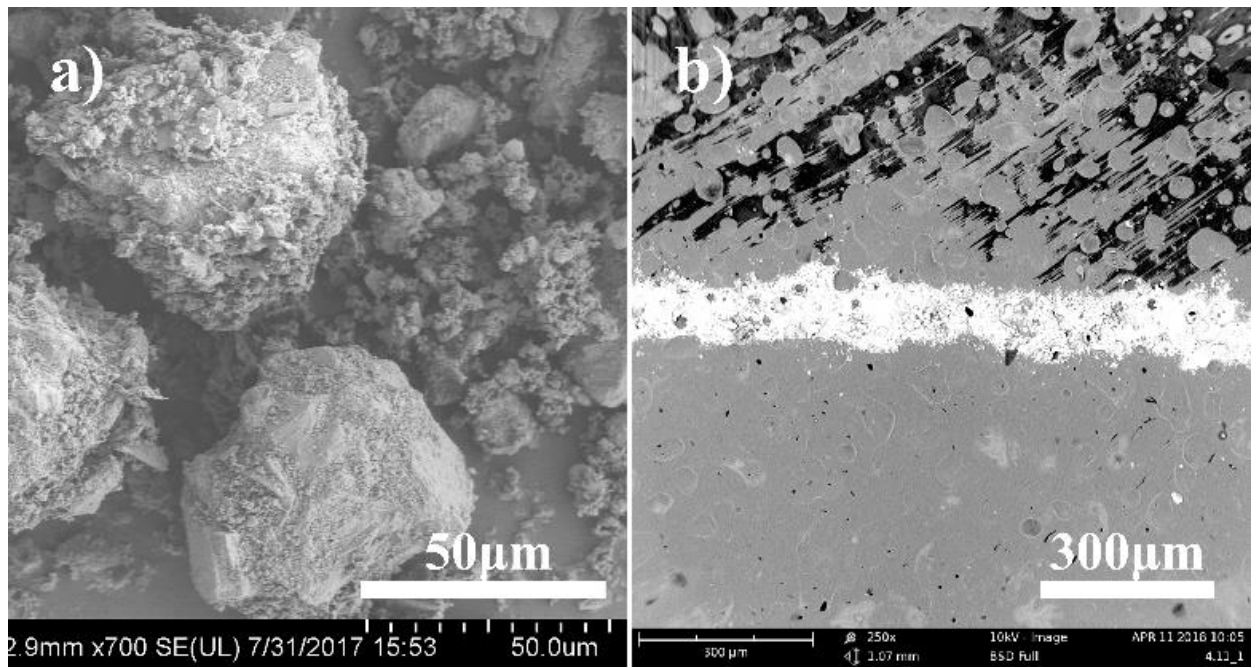

Figure 2. SEM images of spray-dried EDTA-Cu: a) particle morphology, b) electroless plated copper line

Using the spray-dried EDTA-Cu powder as an active ingredient and polyurethane as a matrix, electroless copper plating was conducted and the result is presented in Figure $2 \mathrm{~b}$. It can be seen that only the laser-scanned area is plated with copper, and the copper line shows clear edge and good continuity, i.e., exhibits good copper plating selectivity.

\section{Influence of laser}

Laser activation is the key step in LPKF-LDS, where power, pulse frequency, wavelength, spot size, etc. play important roles in controlling the plating fineness. In order to get a rule, three different types of lasers were used to scan EDTA-Cu modified polyurethane: 1) normal laser marking machine with a wavelength of $1064 \mathrm{~nm}$ (Figs. 1a, 1b and 2)), 2) picosecond laser marking machine with a wavelength of $1064 \mathrm{~nm}$ (Figs. 3a, 4a and 4b) and 3) ultraviolet (UV) laser marking machine with a wavelength of $355 \mathrm{~nm}$ (Fig. 3b).

It can be seen from Figure 3a that the copper line prepared by a picosecond laser is dense and the edge is clear, what resulted from the short heat conducting time of picosecond laser [17]. However, due to the big spot diameter, the width of the plated copper line is still unideal. Figure $3 b$ presents the surface morphology of flexible cathode activated by an UV laser. Due to the short wavelength of UV laser ( $355 \mathrm{~nm}$ ), the laser absorption of modified polyurethane is higher than for 
$1064 \mathrm{~nm}$ laser, so although the UV laser power was only $1 \mathrm{~W}$, the polyurethane-EDTA-Cu was more effectively etched.

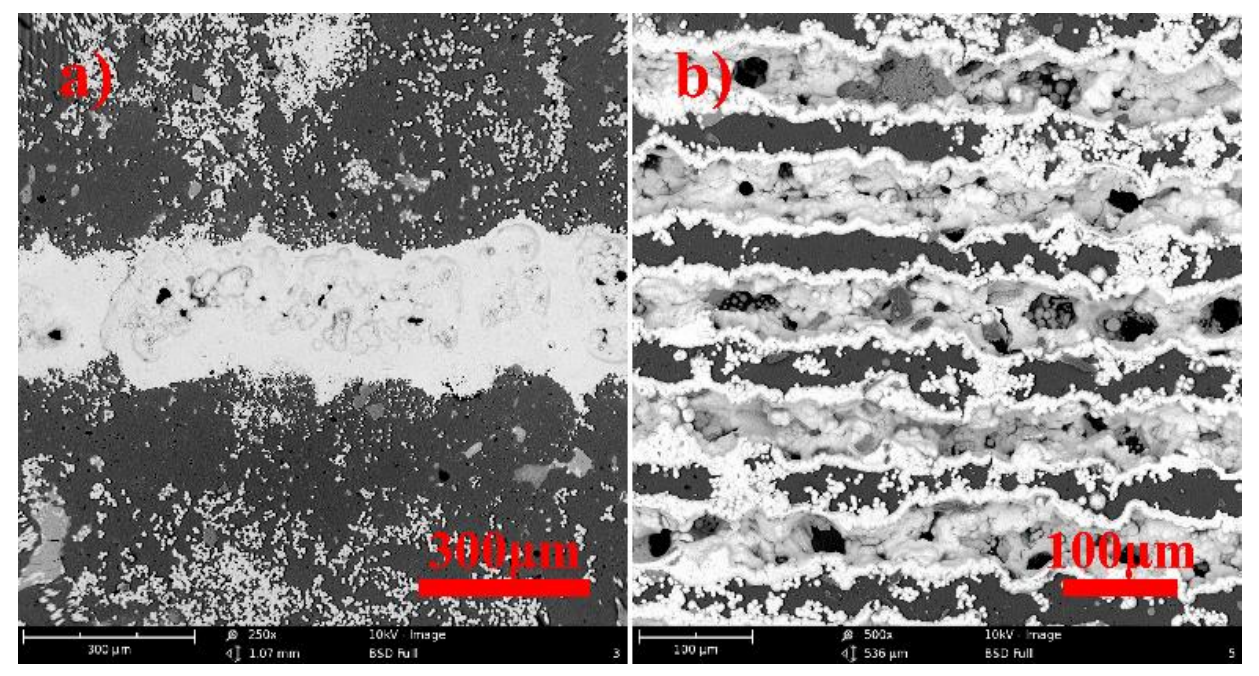

Figure 3. SEM images of flexible cathode scanned by different lasers: a) picosecond laser (1064 nm), b) UV laser (355 nm)

After electroless copper plating, compact and clear copper lines were obtained, showing no mutual influence between copper lines and the line width of about $50 \mu \mathrm{m}$. Specific resistivity was found equal to $\approx 2 \times 10^{-3} \Omega \mathrm{m}$ what is five orders of magnitude higher than for pure $\mathrm{Cu}$. Considering bubbles which cannot be fully eliminated by the antifoaming agent, this high resistivity could result from low continuity and compactness of the plated copper layer.

In order to further improve the surface fineness of electroless copper plating, a small amount of multi-wall carbon nanotubes was added into polyurethane-EDTA-Cu with the mass ratio of EDTA-Cu to carbon nanotubes 10:1. As shown in Figures $4 a$ and $4 b$, the copper lines were denser and clearer than for pure EDTA-Cu samples.

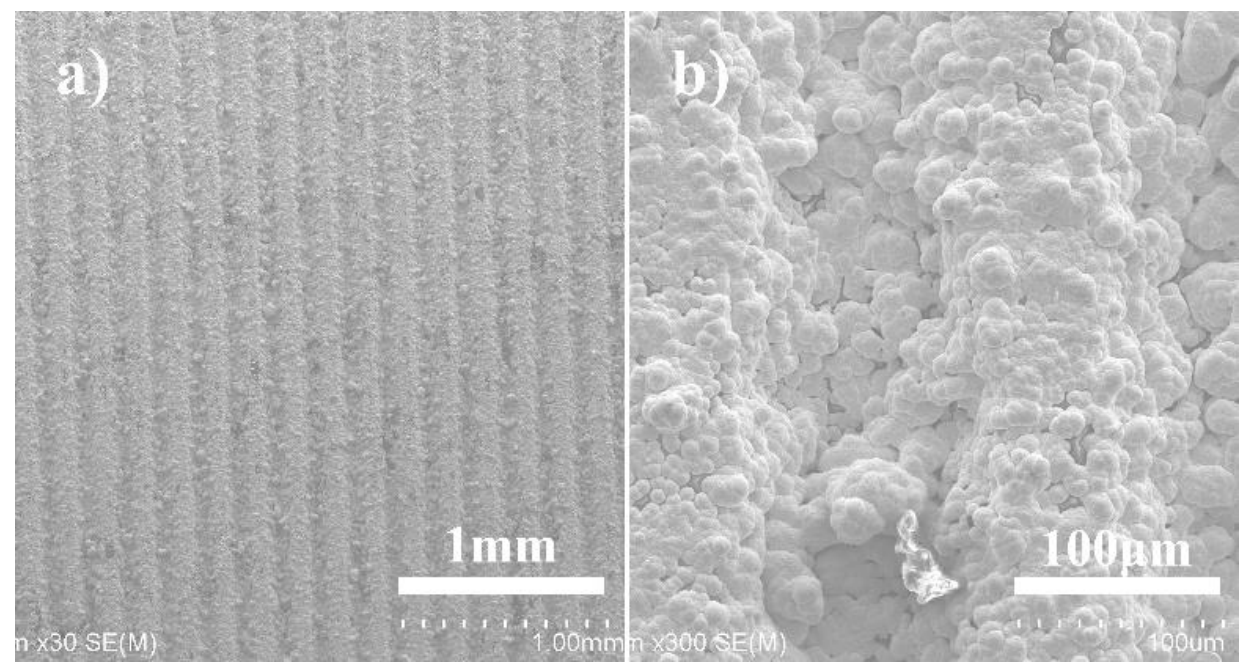

Figure 4. SEM images of flexible cathodes with EDTA-Cu and carbon nanotubes with diameters:

a) $300 \mu \mathrm{m}$, b) $40 \mu \mathrm{m}$

The line width is only $40 \mu \mathrm{m}$, while specific resistivity was determined as $\approx 1.4 \times 10^{-3} \Omega \mathrm{m}$. During the laser scanning, polyurethane was removed and carbon nanotubes were exposed to air. Chemical bond between EDTA and copper ion was broken down and consequently, during the electroless copper plating process, both carbon nanotubes and copper ions act as copper plating seeds, leading to improvement of the surface fineness. 


\section{Electroless copper plating mechanism of EDTA-Cu}

In order to reveal the copper plating mechanism of the flexible cathode with EDTA-Cu, laser activation (scanning) areas were characterized by XPS and XAES. The results are shown in Figures 5 and 6.

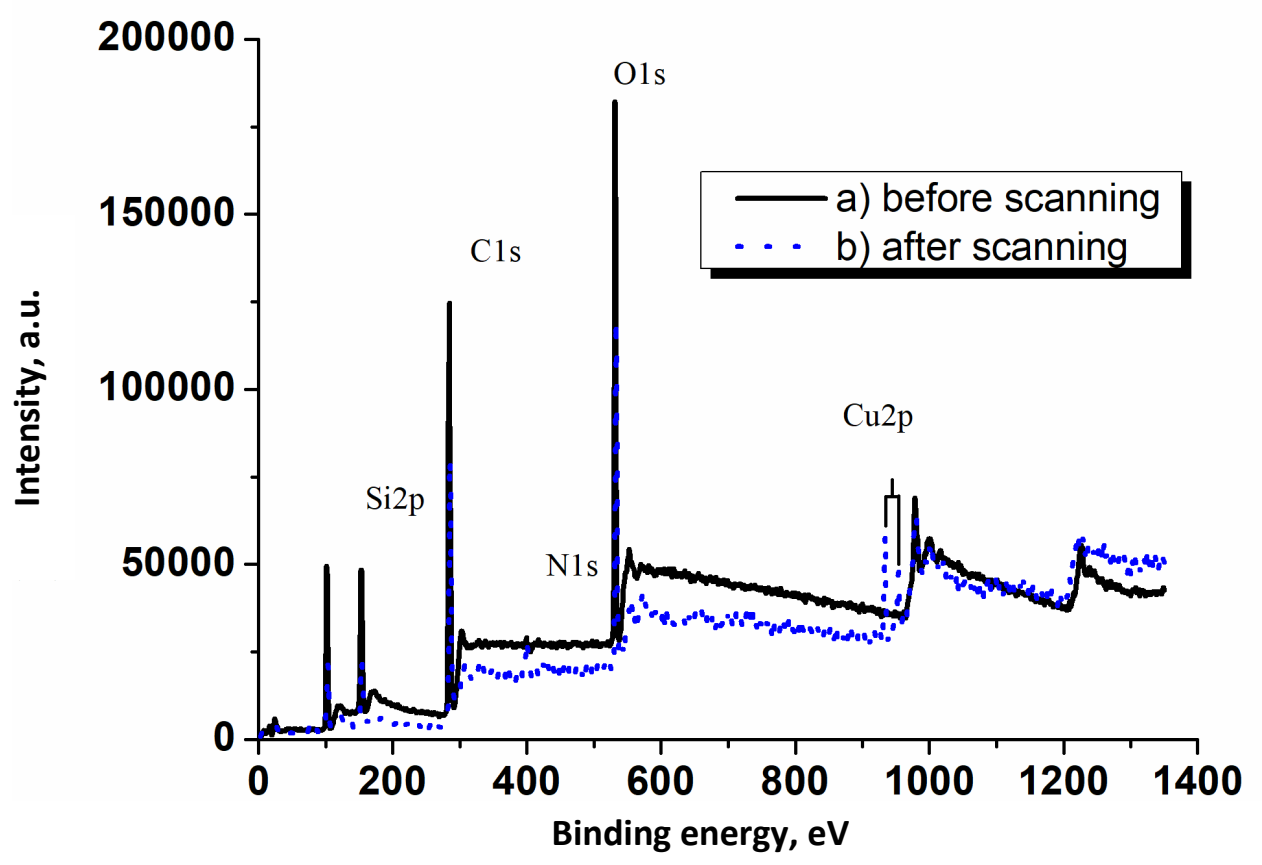

Figure 5. XPS results of the flexible cathode of EDTA-Cu modified polyurethane

Curve a) in Figure 5 shows that no copper was detected before laser scanning, indicating that the surface of the EDTA-Cu modified polyurethane exhibited non-metallic material properties. Obvious Cu2P peak can be identified in Figure 5 curve b) which indicates that copper appeared after laser scanning. During the laser scanning, the polyurethane on the top surface was removed and the chelating bond between copper and EDTA was broken down. As a consequence, Cu particles were exposed and exist in a free form which can play as catalytic sites in the electroless copper plating process [18].

As shown in Figure 6 a), two main peaks can be identified in the laser scanned area, $\mathrm{Cu} 2 \mathrm{p}_{3 / 2}$ $(933.29 \mathrm{eV})$ and $2 \mathrm{p}_{1 / 2}(953.21 \mathrm{eV})$.
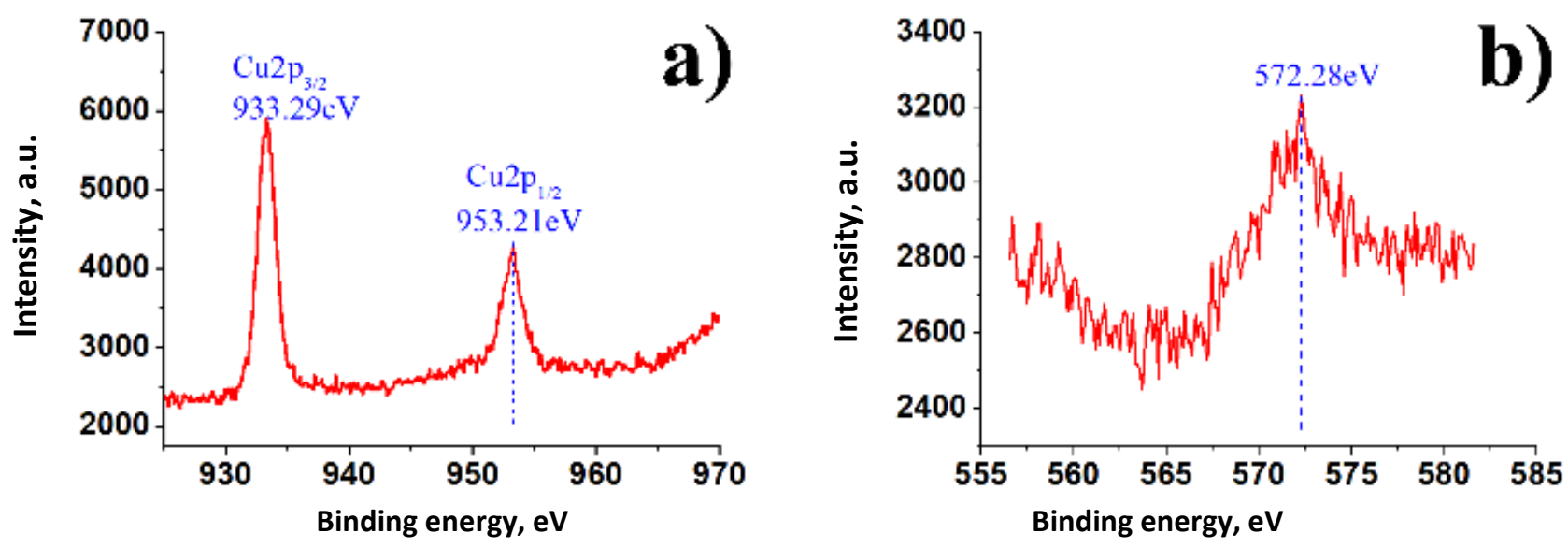

Figure 6. a) XPS spectrum of Cu2p, b) XAES spectrum of CULMM 
No obvious vibrational peak between two peaks indicates that there was no divalent copper in the activation area. The shapes of $\mathrm{Cu}^{0}$ and $\mathrm{Cu}^{+}$peaks are similar to each other and it is difficult to distinguish between them. To identify the specific valence of copper, the laser scanned area was further characterized by XAES [19]. Figure 6b) shows the XAES spectrum of CuLMM (BE=572.28 eV) and kinetic energy as much as $913.8 \mathrm{eV}$ can be obtained according to $\mathrm{KE}=1486.6-\mathrm{BE}(\mathrm{CuLMM})$. The modified Auger parameter can be obtained according to: $\alpha=\mathrm{BE}\left(\mathrm{Cu} 2 \mathrm{p}_{2 / 3}\right)+\mathrm{KE}=1847.09 \mathrm{eV}$. Referring to Auger spectral database, it can be inferred that in the laser scanned area, the copper exists in a form of $\mathrm{Cu}^{+}$[20]. Therefore, it can be confirmed that chemical bonds between EDTA and $\mathrm{Cu}$ were broken down by the laser, and the resultant $\mathrm{Cu}^{+}$acted as a catalytic core in the electroless copper plating.

\section{Adhesion measuring}

Figure 7 shows adhesion measuring results of electroless plated copper at different substrates.

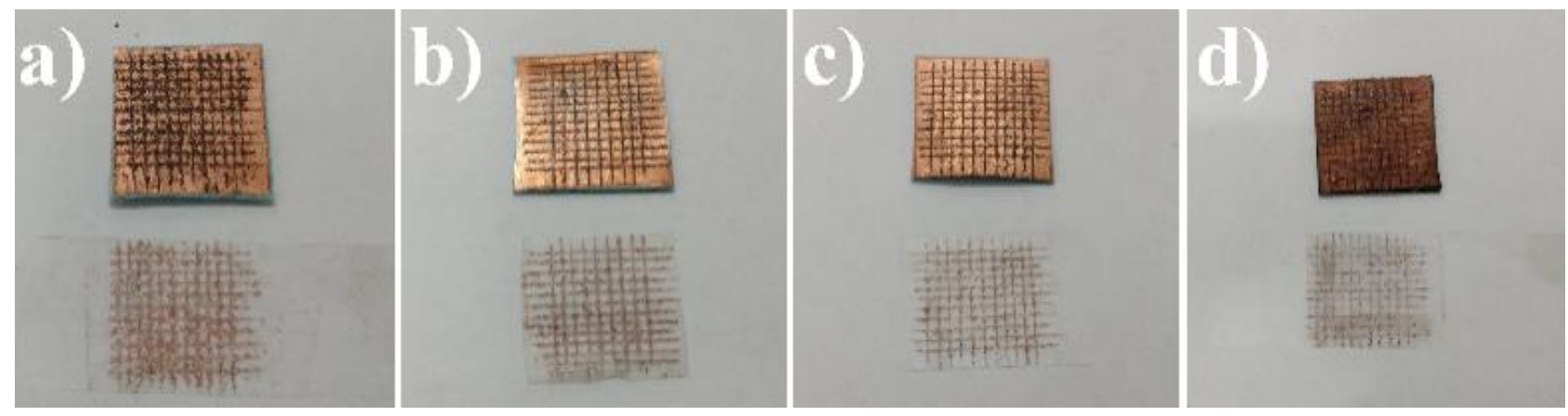

Figure 7. Adhesion measuring results of: a) as-prepared EDTA-Cu, b) suction filtered EDTA-Cu,

c) spray-dried EDTA-Cu, d) EDTA-Cu with carbon nanotubes

According to the norm ASTM-D3359-09, adhesion can be divided into six levels. For Level 5B, the edges of the cuts are completely smooth and none of the squares of the lattice is detached. For Level 4B, small flakes of the coating are detached at intersections and less than $5 \%$ of the area is affected. For Level $3 \mathrm{~B}$, small flakes of the coating are detached along edges and at intersections of cuts and the affected area is 5 to $15 \%$ of the lattice. For Level $2 \mathrm{~B}$, the coating has flaked along the edges and on parts of the squares and the affected area is 15 to $35 \%$ of the lattice. For Level $1 B$, the coating has flaked along the edges of cuts in large ribbons and whole squares have detached. The affected area is 35 to $65 \%$ of the lattice. For Level OB, flaking and detachment is worse than for Level 1B. Through comparing with the standard card, the adhesion strength of the samples can be classified and rated. Carbon nanotubes doped EDTA-Cu (Fig. 7d) and spray-dried (Fig. 7c) EDTA-Cu performed a level 4B, while samples that used suction filtered EDTA-Cu (Fig. 7b) and as-prepared EDTA-Cu (Fig. 7a) can be separately identified as level $3 B$ and $2 B$, respectively. After removing $\mathrm{CH}_{3} \mathrm{COOH}$ and spray drying, spherical EDTA-Cu particles can be evenly distributed into polyurethane and thus performed good adhesion.

\section{Results of electrochemical machining}

Figure 8 shows the electrochemical machining results using the flexible polyurethane-EDTA-Cu as cathode.

As shown in Figure 8, the flexible cathode containing EDTA-Cu can etch clear and neat lines in 5\% sodium nitrate electrolyte on $\mathrm{Zn}$-coated steel. But the width of the etched line is still not ideal, the processing parameters and the flow channel of electrochemical machining need to be further optimized. 


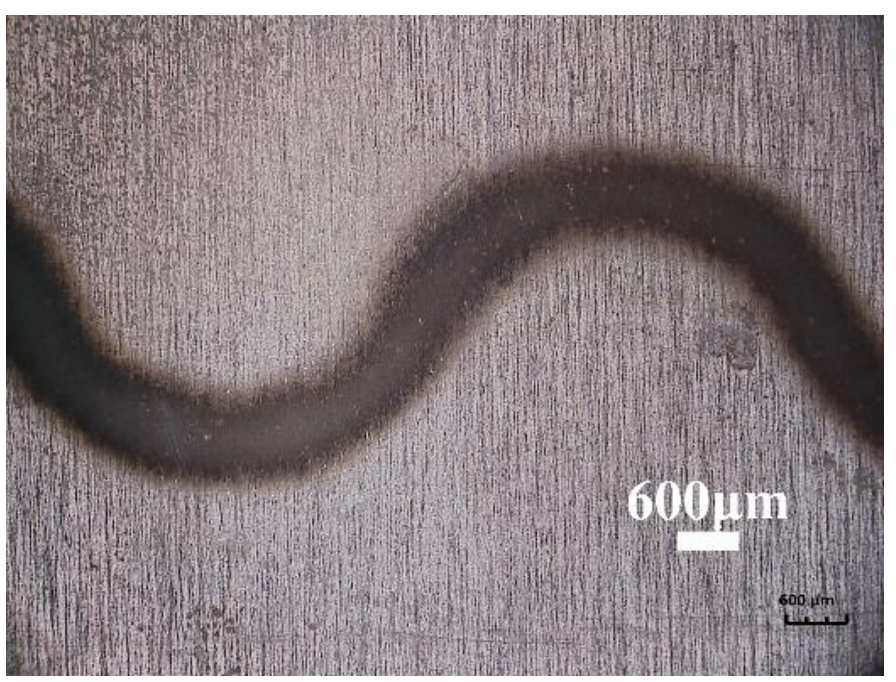

Figure 8. Electrochemical machining sample based on flexible cathode of EDTA-CU and polyurethane

\section{Conclusions}

Using EDTA-Cu as the active material and polyurethane as the matrix, flexible cathodes were prepared by the laser-induced electroless copper plating process. Lasers with different wavelength and frequency were used to scan the EDTA-Cu modified polyurethane, and the polyurethane-EDTA$\mathrm{Cu}$ showed good selectivity in electroless copper plating process. The valence state of copper was investigated and the influence of composition and particle morphology of active material was analyzed.

Experimental results showed that after being scanned by laser, copper is present in the form of $\mathrm{Cu}^{+}$and played a catalytic role in the metallization process. Presence of $\mathrm{CH}_{3} \mathrm{COOH}$, however, played a negative role in the electroless copper plating process. Dissolved in the plating solution, $\mathrm{CH}_{3} \mathrm{COOH}$ initiated formation of many microscopic voids on the surface, what reduced the selectivity of electroless copper plating. The particle morphology of EDTA-Cu was found important through experiments with spray-dried spherical EDTA-Cu particles that were uniformly dispersed in the matrix, improving thus the quality of the plated copper significantly. Properties of the laser also played a significant role on the coating quality. The picosecond laser can produce a dense copper coating, UV laser can be efficiently absorbed by the polyurethane-EDTA-Cu, and the plated copper lines were clear and uniform. Multi-wall carbon nanotubes can also improve the coating fineness, while keeping the flexibility of the material.

In summary, flexible cathodes from EDTA-Cu and polyurethane showed good selectivity for copper plating. Due to additional properties such as low cost and easy preparation, EDTA-Cu is a good choice for flexible cathode electrochemical machining (FCEM).

Acknowledgments: Supported by National Natural Science Foundation of China (51475174), Natural Science Foundation of Fujian Province (2017J01089), Scientific Research Funds of Huaqiao University (Z16Y0020).

\section{References}

1. M. Hüske, J. Kickelhain, J. Müller, G. Eßer, Proc. Lane 8 (2001) 266-270.

2. Yang Li, Bin Liu, Engineering Plastics Application 7 (2013) 100-104.

3. Zhen-Fa Xing, Printed Circuit Information 21 (2003) 37-39.

4. K. C. Yung, C. Chen, C. P. Lee, Applied Surface Science 257 (2011) 6601-6606.

5. Ming Lv, Jiang-guo Liu, Su-huan Wang, Xiao-yan Zeng, Applied Surface Science 366 (2016) 227-232. 
6. Ming Lv, Jiang-guo Liu, Su-huan Wang, Jun Ai, Applied Surface Science 353 (2015) 1150-1155.

7. J. Marques-Hueso, T. D. A. Jones, D. E. Watson, A. Ryspayeva, M. N. Esfahani, M. P. Shuttleworth, R. A. Harris, R. W. Kay, M. P. Y. Desmulliez, Advanced Functional Materials 28 (2018) 1704-1710.

8. J. U. Yang, H. C. Jin, M. J. Yoo, Composites Part B. 110 (2017) 361-367.

9. K. Ratautas, M. Gedvilas, I. Stankeviciene, A. Jagminiene, E. Norkus, G. Raciukaitis, S. Sinopoli, U. Emanuele, N. L. Pira., Congress Molded Interconnect Devices 2016, Molded Interconnect Devices, Würzburg, Germany, 2016, p. 1-5.

10. K. Ratautas, M. Gedvilas, I. Stankevičiene, A. Jagminienė, E. Norkus, N. L. Pira, S. Sinopoli, G. Račiukaitis, Applied Surface Science 412 (2015) 319-326.

11. Y. Farraj, M. Layani, A. Yaverboim, S. Magdassi, Advanced Materials Interfaces 125(2018)1-8.

12. Wen-Juan Cui, Wen-Sheng Lu, Ya-Kun Zhang, Guan-Hua Lin, Tian-Xin Wei, Long Jiang, Colloids \& Surfaces A Physicochemical \& Engineering Aspects 358 (2010) 35-41.

13. Yu-mei Ye, Kai-yong Jiang, Ji-Liang Zhang, Cailiao Kexue Yu Gongyi / Material Science \& Technology 22 (2014) 124-128.

14. Yu-qing Ping, Kai-yong Jiang, Engineering Plastics Application 03 (2015) 54-59.

15. Qi-He Le, Hua Qiao University 41 (2017) 71-76.

16. L. Shi, The Twelfth Annual Meeting of China Polyurethane Industry Association Shanghai, China, 2004, p. 52.

17. D. Bäuerle, Applied Surface Science 186 (2002) 1-6.

18. Meng Wang, Tian Jing University 23 (2011) 19-23.

19. Zheng-Long Wu, Modern Instruments 1 (2009) 58-61.

20. T. Abe, Y. Kashiwaba, M. Baba, J. Imai, H. Sasaki, Applied Surface Science 175 (2001) 549-554.

(C)2018 by the authors; licensee IAPC, Zagreb, Croatia. This article is an open-access article distributed under the terms and conditions of the Creative Commons Attribution license (http://creativecommons.org/licenses/by/4.0/) 